Revista de Matemática: TeORÍA y Aplicaciones 2017 24(1) : 45-60

CIMPA - UCR ISSN: 1409-2433 (PRINT), 2215-3373 (ONLINE)

\title{
EL FENÓMENO DEL ESPÍN SEMIENTERO, CUATERNIOS, Y MATRICES DE PAULI
}

\section{THE PHENOMENON OF HALF-INTEGER SPIN, QUATERNIONS, AND PAULI MATRICES}

\author{
FERnANDO R. GONZÁLEZ-DÍAZ* \\ RICARDO GARCÍA-SALCEDO ${ }^{\dagger}$
}

Received: 3/Mar/2016; Revised: 16/Aug/2016;

Accepted: 28/Oct/2016

\begin{abstract}
Revista de Matemática: Teoría y Aplicaciones is licensed under a Creative Commons Reconocimiento-NoComercial-Compartirigual 4.0 International License.

Creado a partir de la obra en http://www.revistas.ucr.ac.cr/index.php/matematica

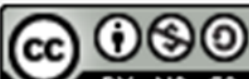

*Escuela Agrícola Panamericana, Zamorano, Valle de Yeguare, F.M., Honduras y CICATA-Legaria, Instituto Politécnico Nacional, México D.F., CP 11500, México. E-Mail: fgonzalez@zamorano.edu

${ }^{\dagger}$ CICATA-Legaria, Instituto Politécnico Nacional, México D.F., CP 11500, México. E-Mail: rigarcias2@ipn.mx
\end{abstract}




\title{
Resumen
}

En este trabajo se reproduce el fenómeno del espín semientero de la ejemplificación que hizo Paul A. M. Dirac con un par de tijeras, una cuerda elástica y una silla. Se describen tres ejemplos más en el que aparece el mismo fenómeno y se relaciona la estructura algebraica de los cuaternios con uno de los ejemplos. Se describen los resultados básicos de las estructuras algebraicas de los cuaternios $\mathbb{H}$, y se establece una relación intrínseca con el fenómeno espín semientero y las matrices de Pauli.

Palabras clave: Espín semientero, Cuaternios, matrices de Pauli, grupo fundamental.

\begin{abstract}
In this paper the phenomenon of half-integer spin exemplification Paul AM Dirac made with a pair of scissors, an elastic cord and chair play. Four examples in which the same phenomenon appears and the algebraic structure of quaternions is related to one of the examples are described.

Mathematical proof of the phenomenon using known topological and algebraic results are explained. The basic results of algebraic structures are described quaternions $\mathbb{H}$, and an intrinsic relationship with the phenomenon half-integer spin and the Pauli matrices is established.
\end{abstract}

Keywords: half-integer spin, quaternions, Pauli matrices, fundamental group.

Mathematics Subject Classification: 97U60, 15A66.

\section{Introducción}

El concepto del espín de las partículas está considerado dentro de los programas de estudio a partir del nivel de educación básica, aunque en este nivel solo se ve como una propiedad de la materia que solo sirve para clasificar a los átomos.

Sin embargo, la comprensión de este concepto es muy complicada e incluso a nivel superior es muy difícil encontrar estudiantes que lo comprendan bien [23], [21]. Particularmente en el nivel superior, la dificultad proviene de la diferencia entre los paradigmas de la mecánica clásica y la mecánica cuántica [13], [8].

La enseñanza de conceptos de la mecánica cuántica en la física o matemática introductorias se ha convertido cada vez más en un tema muy importante debido a las aplicaciones tecnológicas modernas que se basan en ella. Sin embargo, la mayoría de los tratamientos de la teoría cuántica en el nivel introductorio son superficiales, dejando a los estudiantes con la impresión de que la mecánica cuántica no son más que matemáticas abstractas de difícil comprensión [7]. 
Dentro de los trabajos que se han realizado para analizar las preconcepciones y dificultades en el aprendizaje de la mecánica cuántica se encuentran los realizados en Alemania [17], [19], [5], [6], [14], [15], y en Estados Unidos [10], [1], [9]; sin embargo, en el contexto de latinoamérica hacen falta aún muchos trabajos.

Por otro lado, la historia de cómo William Rowan Hamilton descubrió los números cuaternios o cuaterniones, es muy conocida por la forma peculiar en la que ésta sucedió [4], [25]. Resumiendo la idea de como fueron descubiertos y por la relación que el conocía entre los números complejos y la geometría 2dimensional, quiso generalizar la idea durante muchos años, tratando de inventar una álgebra que desempeñara un papel similar en la geometría 3-dimensional. En lenguaje moderno, estaba buscando una álgebra 3-dimensional normada con división. El problema es que no existe ninguna álgebra 3-dimensional normada con división, lo que realmente se necesita es una álgebra 4-dimensional, cuyos elementos son precisamente los números cuaternios.

Desde el punto de vista educativo, los cuaternios se introducen a nivel superior que regularmente se toma en los últimos semestres de carreras como matemática, física o ciertas ingenierías. Sin embargo, en los primeros semestres de dichas carreras, se dan cursos en los que se generalizan los números reales en los conocidos como números complejos. Si uno quiere ir un poco más allá, también se podrían generalizar los números complejos en los llamados cuaternios. Estos números cuaternios tienen algunas relaciones con las matrices y, por tanto, con las rotaciones.

Existen algunos artículos previos en los que realizan un análisis similar al presentado en este trabajo, es decir, dar explicaciones más didácticas sobre los cuaternios y sus relaciones con otros temas fundamentales de la matemática, particularmente aquellos relacionados con la física; por ejemplo, la relación entre las rotaciones y los cuaternios [24], la relación entre las matrices de Pauli, cuaternios y espinores [20], la relación entre el movimiento de un conjunto de objetos con espín y la transformación espinorial inducida (no relativista) escrita por medio de cuaternios [12], relación entre cuaternios y cinturón de Dirac [22]. Sin embargo, en este trabajo se muestra adicionalmente algunas explicaciones al famoso experimento de las tijeras de Dirac y algunos otros relacionados.

Los cuaternios se han vuelto de suma importancia por su utilidad en las representaciones de las rotaciones por ser más compactos y más rápidos de calcular que por otros objetos algebraicos. Su utilidad aparece en temas de física y geometría como estructura vectorial.

En este artículo nos proponemos dar una explicación básica y comprensible de la forma en que se relaciona el famoso experimento de las tijeras de Dirac 
con los cuaternios y sus representaciones. Al mismo tiempo, la forma en la que podría representarse el espín semientero de las partículas elementales desde un punto de vista clásico por medio de algunos experimentos sencillos (incluso pudiera decirse que experimentos caseros debido al tipo de material utilizado), así como por medio de los números cuaternios.

El artículo está organizado de la siguiente manera: en la sección 2 se describen el problema de las tijeras de Dirac. En la sección 3 se describen los resultados algebraicos elementales y dos representaciones matriciales de los cuaternios. En la sección 4 se describe la relación de los cuaternios con el fenómeno del espín semientero y las matrices de Pauli. Finalmente, en la sección 5 se dan las conclusiones.

\section{El truco de las tijeras de Dirac}

Una experiencia de la vida cotidiana que solemos hacer cuando nos encontramos charlando mientras tomamos café, es hacer girar una moneda, el celular, un lápiz, etc. sobre la mesa, de tal manera que cuando gira una vuelta completa, es decir, $2 \pi$ radianes, éste regresa a su posición original. Sin embargo, estamos interesados en mostrar un experimento, que consiste en un objeto particular que al girarlo una vuelta completa, éste no regresa a la misma forma que tenía antes de girarlo y que para que eso suceda, es necesario girarlo dos vueltas completas, es decir, $4 \pi$ radianes.

El experimento del que estamos hablando se denomina el problema de las tijeras de Dirac [16] y se puede construir con material fácil de conseguir. Se requieren unas tijeras, dos cuerdas elásticas de $40 \mathrm{~cm}$ y un poste de madera de $30 \mathrm{~cm}$.

Para construir el objeto del experimento, hacemos lo siguiente: un extremo de cada cuerda lo atamos en cada uno de los agujeros de las tijeras, después el otro extremo de las cuerdas lo atamos con una separación de $7 \mathrm{~cm}$ al poste previamente fijado en forma horizontal, como se muestra en la figura 1-A. El objeto del experimento son las tijeras y las cuerdas atadas como se menciono anteriormente. Sujetando las tijeras con la mano y manteniendo las cuerdas estiradas, le denominaremos posición inicial o trivial del objeto y lo denotamos por $C_{0}$, como se muestra en la figura 1-A.

Ahora, comenzamos el experimento teniendo el objeto en su posición inicial, posteriormente las tijeras se rotan alrededor de su eje de simetría una vuelta completa ( $2 \pi$ radianes) y observamos que las cuerdas se enredan, a esta posición la denotamos por $C_{1}$, como se muestra en la figura 1-B. Claramente las tijeras vuelven a su estado original, pero las cuerdas no, ya que éstas se enredaron. 


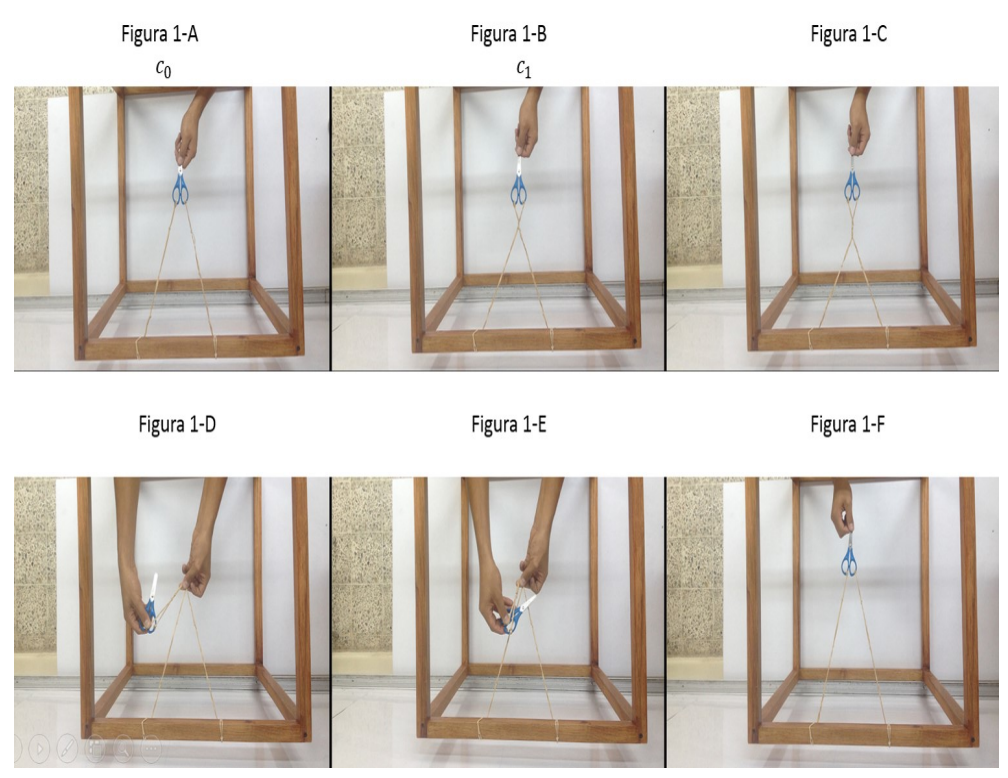

Figura 1: Disposición del experimento de las tijeras de Dirac.

De esta forma, como el objeto es el conjunto tijeras-cuerdas, se puede decir que el no ha regresado a su posición original después de una vuelta completa.

Teniendo el objeto en la posición, $C_{1}$ (con las cuerdas enredadas), intentemos desenredar las cuerdas, estirándolas y manipulándolas pero conservando la posición y dirección de las tijeras respecto del poste fijo. Sin embargo, no importa la maniobra, simple o complicada que se intente, las cuerdas no regresan a su estado original $C_{0}$, es decir, desenredadas.

Coloquemos nuevamente el objeto en su posición original $C_{0}$. Ahora rotamos las tijeras alrededor de su eje de simetría dos vueltas completas, es decir, $4 \pi$ radianes. Observamos que las cuerdas parecen estar más enredadas que en el procedimiento anterior, como se muestra en la figura 1-C. ¿Habrá alguna forma de desenredar las cuerdas conservando la posición y dirección de las tijeras respecto del poste fijo? La respuesta es que sí.

El procedimiento para desenredar las cuerdas es el siguiente: Sujetamos las cuerdas por el medio de su longitud y las estiramos hasta la punta de las tijeras, como se muestra en la figura 1-D. Manteniendo la dirección y posición de las tijeras pasamos las tijeras por debajo de las cuerdas (figura 1-E), al soltarlas, nos llevaremos la sorpresa de que las cuerdas regresan también a su estado original $C_{0}$, figura 1-F, es decir, se desenredan. Por lo que el objeto, conjunto tijerascuerdas, han regresado a su posición original $C_{0}$. 
En general, podemos girar un número impar de vueltas completas al objeto y al intentar desenredar las cuerdas nos será imposible. Pero si rotamos las tijeras un número par de vueltas completas lograremos desenredar las cuerdas.

Existe una variante de este experimento en la que solo se utiliza una carta de una baraja y un listón de tres o cuatro centímetros de ancho ${ }^{1}$. Finalmente, existe otra actividad semejante conocida como la llave espinorial, la cual está descrita en [2]. Cada una de éstas actividades aunque similares, se pueden realizar en dependencia de los materiales con los que se cuente.

En cada uno de los experimentos anteriores, ocurre un fenómeno diferente al estado del sistema, ya sea que se haya rotado $2 \pi$ radianes o $4 \pi$ radianes. En cada caso, el sistema es más que sólo un par aislado de tijeras, en realidad son un par conectado con el medio, es decir, tijera-cuerda, carta-listón, llave-cuerdas, respectivamente.

\section{3 Álgebra de los cuaternios $\mathbb{H}$}

En esta sección trataremos de dar una explicación desde el punto de vista matemático al fenómeno que se presenta en los experimentos de la sección anterior.

El campo de los números reales $\mathbb{R}$ corresponde con todas aquellas magnitudes escalares con las que trabajamos cotidianamente. Una generalización de este campo es el de los llamados números complejos $\mathbb{C}$, los cuales están formados como la combinación lineal de un número real $\mathbb{R}$ y un número imaginario, el cual consiste de un múltiplo del número $i$ definido como $i^{2}=-1$, de tal forma que los números complejos se definen como:

$$
\mathbb{C}=\left\{z=a+b i\left|i^{2}=-1\right| a, b, \in \mathbb{R}\right\} .
$$

La siguiente generalización de números corresponde a los conocidos como cuaternios o cuaterniones, los cuales forman un espacio métrico como los números $\mathbb{R}$. Esto lo describimos en la siguiente subsección.

\subsection{Estructura algebraica básica de los cuaternios}

Consideremos el campo de los números reales $\mathbb{R}$, definimos el conjunto de los cuaternios como:

$$
\begin{aligned}
& \mathbb{H}=\left\{q=a+b i+c j+d k\left|i^{2}=j^{2}=k^{2}=i j k=-1\right| a, b, c, d \in \mathbb{R}\right\}, \\
& { }^{1} \text { http: //www. wetsavannaanimals. net/wordpress/tag/ } \\
& \text { dirac-belt-trick/ }
\end{aligned}
$$


y la suma de dos elementos en este conjunto está dada por la siguiente regla:

$$
\begin{aligned}
q_{1}+q_{2} & =(a+b i+c j+d k)+(p+r i+s j+t k) \\
& =(a+p)+(b+r) i+(c+s) j+(d+t) k .
\end{aligned}
$$

El producto escalar de un número real por un cuaternio cumple la siguiente regla:

$$
r q_{1}=r(a+b i+c j+d k)=r a+r b i+r c j+r d k .
$$

Con estas operaciones los cuaternios forman un $\mathbb{R}$-espacio vectorial de dimensión 4, siendo isomorfo a $\mathbb{R}^{4}$ con base, $\{1, i, j, k\}$.

Haciendo una analogía a los números complejos $\mathbb{C}$, un cuaternio tiene una parte real, $\operatorname{Re}(q)=a$ y una parte imaginaria, $\operatorname{Im}(q)=b i+c j+d k$. La relación de la multiplicación $i^{2}=j^{2}=k^{2}=i j k=-1$ y las permutaciones cíclicas, $i j=k=-j i$, determinan todos los posibles productos para los elementos de la base. Las relaciones de la multiplicación nos permiten definir el producto de dos cuaternios por la regla:

$$
\begin{aligned}
q_{1} q_{2} & =(a+b i+c j+d k)(p+m i+r j+s k) \\
& =(a p-b m-c r-d s)+(a m+b p+c s-d r) i+ \\
& +(a r-b s+c p+d m) j+(a s+b r-c m+d p) k .
\end{aligned}
$$

A diferencia de la multiplicación de los números complejos o reales, la multiplicación de los cuaternios no es conmutativa. La operación conjugación de los cuaternios es análoga a la de los números complejos, es decir, para un cuaternio $q=a+b i+c j+d k$ su conjugado está dado por $\bar{q}=a-b i-c j-d k \mathrm{y}$ nos permite definir la norma o magnitud

$$
q \bar{q}=|q|^{2}=a^{2}+b^{2}+c^{2}+d^{2} .
$$

Por otro lado, cualquier cuaternio distinto de cero tiene un inverso multiplicativo definido por $q^{-1}=\frac{\bar{q}}{|q|^{2}}$. Estas operaciones le dan a los cuaternios una estructura de álgebra normada con división. La norma anterior nos ayuda a definir la distancia entre dos cuaternios, $d\left(q_{1}, q_{2}\right)=\left|q_{2}-q_{1}\right|$, lo que nos indica que los cuaternios forman un espacio métrico. 


\subsection{Forma matricial de los cuaternios}

Existen al menos dos formas de representar a los cuaternios $\mathbb{H}$ como matrices, de tal manera que su suma y producto correspondan a la suma y producto usual de matrices en $\mathbb{R}[3]$.

La razón por la que vamos a representar a los cuaternios como matrices es para poder realizar una identificación entre estos números con las llamadas matrices de Pauli, que son aquellas que, desde el punto de vista físico, describen el espín en las partículas elementales [26].

Para construir la primera forma matricial, definimos el homomorfismo inyectivo $^{2}$ de álgebra $f: \mathbb{H} \rightarrow M_{2}(\mathbb{C})$ del álgebra de los cuaternios $\mathbb{H}$, al álgebra de matrices de $2 \times 2$ con entradas complejas, denotado por $M_{2}(\mathbb{C})$. Definido por la siguiente regla de correspondencia:

$$
f(q) \equiv f(a+b i+c j+d k)=\left(\begin{array}{cc}
a+b i & c+d i \\
-c+d i & a-b i
\end{array}\right) \equiv \mathbf{A} .
$$

La aplicación de la función o morfismo $f$ los números $1, i, j, k$, nos da como resultado las siguientes matrices:

$$
\begin{gathered}
f(1)=\left(\begin{array}{cc}
1 & 0 \\
0 & 1
\end{array}\right) \equiv \mathbf{E}_{1}, f(i)=\left(\begin{array}{cc}
i & 0 \\
0 & -i
\end{array}\right) \equiv \mathbf{I}_{1}, \\
f(j)=\left(\begin{array}{cc}
0 & 1 \\
-1 & 0
\end{array}\right) \equiv \mathbf{J}_{1}, f(k)=\left(\begin{array}{cc}
0 & i \\
i & 0
\end{array}\right) \equiv \mathbf{K}_{1} .
\end{gathered}
$$

La matriz A se puede escribir como una combinación lineal, de las matrices anteriores de la siguiente forma:

$$
\mathbf{A}=a \mathbf{E}_{1}+b \mathbf{I}_{1}+c \mathbf{J}_{1}+d \mathbf{K}_{1}
$$

La segunda forma matricial de los cuaternios se construye con el homomorfismo inyectivo de álgebras

$$
g: \mathbb{H} \rightarrow M_{4}(\mathbb{R})
$$

del álgebra de los cuaternios, al álgebra de matrices $(4 \times 4)$ reales, definido por:

\footnotetext{
${ }^{2}$ Un homomorfismo que va de un objeto matemático del conjunto $X$ a otro $Y$ con la misma estructura algebraica, es una función que preserva las operaciones definidas en dichos objetos. Se dice inyectivo porque en el conjunto $X$ no puede haber dos o más elementos que tengan la misma imagen en $Y$.
} 


$$
g(a+b i+c j+d k)=\left(\begin{array}{cccc}
a & b & c & d \\
-b & a & -d & c \\
-c & d & a & -b \\
-d & -c & b & a
\end{array}\right) \equiv \mathbf{C} .
$$

La aplicación del morfismo $g$ sobre los números $1, i, j, k$, nos da como resultado las siguientes matrices:

$$
\begin{gathered}
g(1)=\left(\begin{array}{cccc}
1 & 0 & 0 & 0 \\
0 & 1 & 0 & 0 \\
0 & 0 & 1 & 0 \\
0 & 0 & 0 & 1
\end{array}\right) \equiv \mathbf{E}_{2}, \\
g(i)=\left(\begin{array}{cccc}
0 & 1 & 0 & 0 \\
-1 & 0 & 0 & 0 \\
0 & 0 & 0 & -1 \\
0 & 0 & 1 & 0
\end{array}\right) \equiv \mathbf{I}_{2}, \\
g(j)=\left(\begin{array}{cccc}
0 & 0 & 1 & 0 \\
0 & 0 & 0 & 1 \\
-1 & 0 & 0 & 0 \\
0 & -1 & 0 & 0
\end{array}\right) \equiv \mathbf{J}_{2}, \\
g(k)=\left(\begin{array}{cccc}
0 & 0 & 0 & 1 \\
0 & 0 & -1 & 0 \\
0 & 1 & 0 & 0 \\
-1 & 0 & 0 & 0
\end{array}\right) \equiv \mathbf{K}_{2} .
\end{gathered}
$$

De esta forma, la matriz $C$ se puede escribir como una combinación lineal de las matrices anteriores como sigue:

$$
\mathbf{C}=a \mathbf{E}_{2}+b \mathbf{I}_{2}+c \mathbf{J}_{2}+d \mathbf{K}_{2}
$$

\section{Aplicación de los cuaternios $\mathbb{H}$ en la Física}

\subsection{Los cuaternios y el espín semientero}

Las relaciones de la estructura algebraica de los cuaternios se pueden identificar con los movimientos de rotación de un disco con cuerdas, al cual le llamamos títere, [11], como se muestra en la Figura 2. 


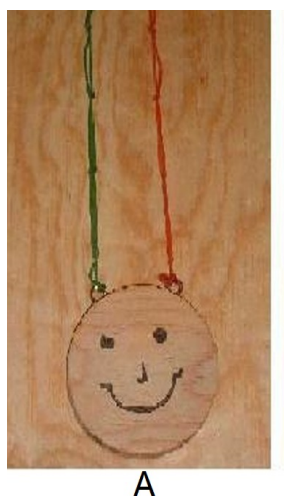

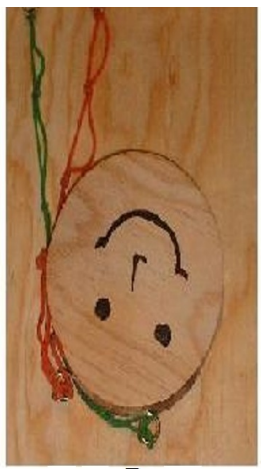

B

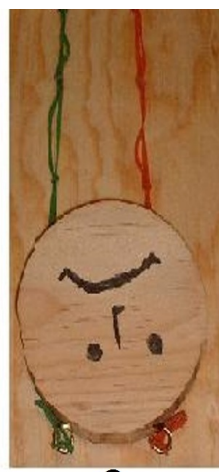

C

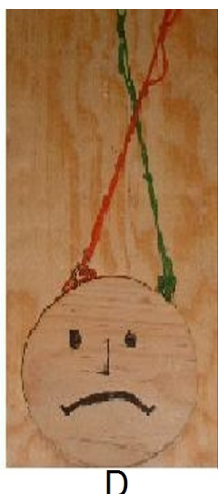

D

Figura 2: Movimientos de rotación de un títere.

El disco sin las cuerdas representa al 4-grupo de Klein ${ }^{3}$, que esta formado por el conjunto

$$
\mathbf{V}=\{\mathbf{E}, \mathbf{I}, \mathbf{J}, \mathbf{K}\}
$$

y su operación algebraica se describe en la siguiente tabla:

\begin{tabular}{|c|c|c|c|c|}
\hline$\cdot$ & $\mathbf{E}$ & $\mathbf{I}$ & $\mathbf{J}$ & $\mathbf{K}$ \\
\hline $\mathbf{E}$ & $\mathbf{E}$ & $\mathbf{I}$ & $\mathbf{J}$ & $\mathbf{K}$ \\
\hline $\mathbf{I}$ & $\mathbf{I}$ & $\mathbf{E}$ & $\mathbf{K}$ & $\mathbf{J}$ \\
\hline $\mathbf{J}$ & $\mathbf{J}$ & $\mathbf{K}$ & $\mathbf{E}$ & $\mathbf{I}$ \\
\hline $\mathbf{K}$ & $\mathbf{K}$ & $\mathbf{J}$ & $\mathbf{I}$ & $\mathbf{E}$ \\
\hline
\end{tabular}

Dicho de otra manera, el 4-grupo de Klein cumple las relaciones: $\mathbf{I}^{2}=\mathbf{J}^{2}=$ $\mathbf{K}^{2}=\mathbf{E}, \mathbf{I J}=\mathbf{J I}=\mathbf{K}$, donde $\mathbf{E}$ es el elemento identidad.

La forma en que se identifica el disco con el 4-grupo de Klein es la siguiente: Consideremos que se dibuja en uno de los lados del disco una cara feliz y en el otro lado del disco una cara triste, dispuesto de la forma en que se muestra en la figura 2. Identificamos a $\mathbf{E}$ con el lado del disco que contiene la cara feliz, figura 2-A, (posición inicial); I con la rotación del disco en la desde la posición inicial un valor de $\pi$ radianes alrededor del eje perpendicular a esta página, ver figura 2-B; J con la rotación del disco partiendo de la posición inicial hasta un valor de $\pi$ radianes alrededor del eje horizontal, figura 2-C; y, finalmente, $\mathbf{K}$ con la rotación del disco partiendo de la posición inicial hasta un valor de $\pi$ radianes alrededor del eje vertical, figura 2-D.

\footnotetext{
${ }^{3}$ Este grupo está formado por cuatro elementos, donde cada elemento es inverso de sí mismo. Para saber más, se puede revisar [18].
} 
Es fácil ver que las identificaciones de los elementos del 4-grupo de Klein con el disco cumplen las operaciones del álgebra de los cuaternios, descritas en la sección anterior. Es decir, al aplicar una rotación par de $\pi$ radianes alrededor de cada uno de los ejes antes mencionados, volvemos a la posición inicial y al aplicarle una rotación $\pi$ radianes alrededor del eje perpendicular a esta página y después $\pi$ radianes alrededor del eje horizontal (o viceversa) obtenemos la rotación asignada a K. Si ahora atamos unas cuerdas al disco, obtenemos lo que llamamos el títere, el cual al aplicarle una rotación de $2 \pi$ radianes, las cuerdas se enredan.

1. Identificación de los cuaternios con los valores del 4-grupo de Klein:

$$
1 \longmapsto \mathbf{E}, i \longmapsto \mathbf{I}, j \longmapsto \mathbf{J}, k \longmapsto \mathbf{K},
$$

2. Identificación de los cuaternios con las matrices obtenidas del homomorfismo $g$, de la segunda forma matricial:

$$
1 \longmapsto \mathbf{E}_{2}, i \longmapsto \mathbf{I}_{2}, j \longmapsto \mathbf{J}_{2}, k \longmapsto \mathbf{K}_{2},
$$

Por lo tanto, considerando las identificaciones equivalentes anteriores de los cuaternios con las rotaciones espaciales aplicadas al títere, al aplicar las rotaciones $i^{2}, j^{2}$ y $k^{2}$ (elevar al cuadrado significa aplicar la rotación correspondiente dos veces) al títere, se hace una torsión de $2 \pi$ radianes en las cuerdas.

De manera análoga se cumple para las matrices $\left(\mathbf{I}_{2}\right)^{2},\left(\mathbf{J}_{2}\right)^{2}$ y $\left(\mathbf{K}_{2}\right)^{2}$, es decir, si identificamos la torsión de $\pm 2 \pi$ radianes de las cuerdas con -1 , obtenemos las igualdades $i^{2}=j^{2}=k^{2}=-1,\left(\mathbf{I}_{2}\right)^{2}=\left(\mathbf{J}_{2}\right)^{2}=\left(\mathbf{K}_{2}\right)^{2}=-\mathbf{E}_{2}$.

Ahora, si aplicamos las correspondientes torsiones una vez más en las cuerdas del títere, se obtienen las igualdades:

$$
\begin{aligned}
1 & =\left(i^{2}\right)^{2}=\left(j^{2}\right)^{2}=\left(k^{2}\right)^{2}, \\
\mathbf{E}_{2} & =\left(\left(\mathbf{I}_{2}\right)^{2}\right)^{2}=\left(\left(\mathbf{J}_{2}\right)^{2}\right)^{2}=\left(\left(\mathbf{K}_{2}\right)^{2}\right)^{2}
\end{aligned}
$$

en otras palabras, las cuerdas del títere regresan a su estado original al aplicarle una rotación de $4 \pi$ radianes, obteniendo el fenómeno del problema de las tijeras de Dirac.

Si una torsión de $\pm 2 \pi$ radianes la identificamos con -1 , entonces podemos identificar la torsión de $\pm \pi$ radianes con $\sqrt{-1}$ y, por consiguiente, a los valores $i, j, k$ y a las matrices $\mathbf{I}_{2}=\mathbf{J}_{2}=\mathbf{K}_{2}$.

Notemos que los conjugados cumplen las igualdades $\bar{i}=-i, \bar{j}=-j, \bar{k}=$ $-k$, el conjugado de los cuaternios puros es equivalente a invertir la rotación 
correspondiente, obteniendo así un cambio de torsión o un aumento en la torsión de $2 \pi$ radianes en las cuerdas.

El fenómeno de los conjugados en los cuaternios es equivalente a las matrices transpuestas, es decir, se cumplen las igualdades:

$$
\left.\left(\mathbf{I}_{2}\right)^{T}=-\mathbf{I}_{2},\left(\mathbf{J}_{2}\right)^{T}=-\mathbf{J}_{2}\right),\left(\mathbf{K}_{2}\right)^{T}=-\mathbf{K}_{2} .
$$

Las relaciones de multiplicación $i j=k, j k=i, k i=j$ y $j i=-k, i k=$ $-j, k j=-i$ de los cuaternios, así como el producto equivalente de las matrices

$$
\begin{aligned}
& \mathbf{I}_{2} \mathbf{J}_{2}=\mathbf{K}_{2}, \mathbf{J}_{2} \mathbf{K}_{2}=\mathbf{I}_{2}, \mathbf{K}_{2} \mathbf{I}_{2}=\mathbf{J}_{2}, \\
& \mathbf{J}_{2} \mathbf{I}_{2}=\left(\mathbf{K}_{2}\right)^{T}, \mathbf{K}_{2} \mathbf{J}_{2}=\left(\mathbf{I}_{2}\right)^{T}, \mathbf{I}_{2} \mathbf{K}_{2}=\left(\mathbf{J}_{2}\right)^{T},
\end{aligned}
$$

también se cumplen en la aplicación de las rotaciones en el títere.

Por lo tanto, el títere, con la identificación de las rotaciones espaciales antes mencionadas, une la estructura algebraica de los cuaterniones y de las matrices obtenidas por el homomorfismo $g$ con el fenómeno del espín semientero que se presenta en las partículas elementales.

Esta es una forma interesante y atractiva de introducir, ya sea el álgebra de los cuaternios o el concepto de espín de la mecánica cuántica. Además de ver una relación directa de una teoría matemática que no se da comúnmente en los cursos universitarios con un fenómeno, el del espín $1 / 2$ que debe verse en cualquier licenciatura que tenga un curso de mecánica cuántica.

\subsection{Los cuaternios y las matrices de Pauli}

En la mecánica cuántica, las matrices de Pauli se encuentran presentes en la llamada ecuación de Pauli que toma en cuenta la interacción de la rotación de una partícula con un campo electromagnético externo.

Cada matriz de Pauli es hermitiana ${ }^{4}$, y junto con la matriz de identidad $\mathbf{E}$, las matrices de Pauli (multiplicado por coeficientes reales) generan el espacio de vectorial completo de matrices de $2 \times 2$ hermitianas.

En el lenguaje de la mecánica cuántica, toda matriz hermitiana es una observable física, por lo que las matrices de Pauli generan el espacio de observables del espacio de Hilbert complejo de 2 dimensiones.

Existe una forma de relacionar a los números cuaternios $\mathbb{H}$ con las matrices de Pauli. Cada matriz de Pauli está relacionada con un operador de momento angular, el cual puede visualizarse clásicamente como las rotaciones de las partículas elementales de espín $1 / 2$, en cada una de las tres direcciones espaciales.

\footnotetext{
${ }^{4}$ Una matriz A hermitiana es una matriz cuadrada cuyos elementos son números, $\mathbb{H}$, que tiene la característica de ser igual a su propia traspuesta conjugada, $\mathbf{A}=\overline{\mathbf{A}}^{T}$.
} 
Para construir esta relación intrínseca, definimos el conjunto de las matrices de Lorentz de la siguiente manera:

$\mathbb{M L}=\left\{L \in M_{2}(\mathbb{C})\right.$

$$
\begin{aligned}
\mid L & =\left(\begin{array}{cc}
a+d & b-i c \\
b+i c & a-d
\end{array}\right) \\
\mid \quad L & \left.=\bar{L}^{T}, \quad \operatorname{det}(L)=a^{2}-b^{2}-c^{2}-d^{2}\right\} .
\end{aligned}
$$

Y la transformación $h: \mathbb{R}^{4} \rightarrow \mathbb{M L}$ que va del espacio vectorial $\mathbb{R}^{4}$ a estas matrices, definida por la siguiente regla de correspondencia:

$$
h(a, b, c, d)=\left(\begin{array}{cc}
a+d & b-i c \\
b+i c & a-d
\end{array}\right)
$$

Al aplicar la transformación $h$ en los valores de la base canónica $\left\{e_{0}=\right.$ $\left.(1,0,0,0), e_{1}=(0,1,0,0), e_{2}=(0,0,1,0), e_{3}=(0,0,0,1)\right\}$ de $\mathbb{R}^{4}$, se obtiene los siguientes resultados:

$$
\begin{gathered}
h\left(e_{0}\right)=\left(\begin{array}{ll}
1 & 0 \\
0 & 1
\end{array}\right):=\sigma_{0}, \quad h\left(e_{1}\right)=\left(\begin{array}{cc}
0 & 1 \\
1 & 0
\end{array}\right):=\sigma_{1}, \\
h\left(e_{2}\right)=\left(\begin{array}{cc}
0 & -i \\
i & 0
\end{array}\right):=\sigma_{2}, \quad h\left(e_{3}\right)=\left(\begin{array}{cc}
1 & 0 \\
0 & -1
\end{array}\right):=\sigma_{3} .
\end{gathered}
$$

Las matrices $\sigma_{1}, \sigma_{2}$ y $\sigma_{3}$ reciben el nombre de las matrices de Pauli, mientras que $\sigma_{0}$ es la matriz identidad. Ahora podemos relacionar los cuaternios y las matrices de Pauli bajo la siguiente relación:

$$
1 \mapsto \sigma_{0}, \quad i \mapsto-i \sigma_{1}, \quad j \mapsto-i \sigma_{2}, \quad k \mapsto-i \sigma_{3}
$$

Esta relación cumple con las operaciones de los cuaternios, es decir:

$$
\begin{aligned}
-1 & =i^{2}=j^{2}=k^{2}=i j k \\
-\sigma_{0} & =\left(-i \sigma_{1}\right)^{2}=\left(-i \sigma_{2}\right)^{2} \\
& =\left(-i \sigma_{3}\right)^{2}=\left(-i \sigma_{1}\right)\left(-i \sigma_{2}\right)\left(-i \sigma_{3}\right) .
\end{aligned}
$$

\section{Conclusiones}

Se presenta una actividad experimental con material de bajo costo que puede ser utilizada en los cursos universitarios de mecánica cuántica o de matemáticas superiores para relacionar conceptos matemáticos como los cuaternios, matrices 
de Pauli y transformaciones de Lorentz con conceptos físicos como el espín de las partículas elementales.

Los cuaternios son una generalización de los números complejos que poseen una estructura de álgebra, lo que permite relacionarlos con otras álgebras más conocidas. En particular, los cuaternios $\mathbb{H}$ se pueden identificar con estructuras algebraicas muy conocidas del álgebra lineal, como lo son las matrices. De esta forma, las operaciones de suma y producto de los cuaternios se conservan.

Las relaciones de la estructura algebraica de los cuaternios se pueden identificar con los movimientos de rotación de un disco con cuerdas. Las matrices que se obtienen al aplicar el homomorfismo $f$ de la primera forma matricial de los cuaternios, $\mathbf{E}_{1}, \mathbf{I}_{1}, \mathbf{J}_{1}, \mathbf{K}_{1}$ cumplen las mismas relaciones que se obtienen por el homomorfismo $g$ de la segunda forma matricial. Por lo tanto, existen al menos dos formas de identificar las operaciones algebraicas de cuaternios y matrices con el fenómeno del espín semientero.

\section{Reconocimientos}

Dedicado a Fernando González Romero.

FRGD agradece a la Dra. Claudia García, directora del departamento de Currículo General de la Escuela Agrícola Panamericana, Zamorano. Este trabajo fue apoyado parcialmente por los proyectos del IPN SIP20160512, SIP20150188. RGS agradece el apoyo de las becas COFAA-IPN, EDI-IPN y SNI-CONACYT.

\section{Referencias}

[1] Bao, L.; Redish, E.F.; Steinberg, R.N. (s.f.) "Student misunderstandings of the quantum wavefunction" (unpublished).

[2] Díaz, F.R.G.; Rodríguez, G.R.M.; Puebla, E.L.; García-Salcedo, R. (2007) "La topología del truco de las tijeras de Dirac", CIENCIA ergo-sum 14(1): 107-112.

[3] Dick, J.; Childrey, M. (2012) "Enhancing understanding of transformation matrices", Mathematics Teacher 105(8): 622-626.

[4] Ebbinghaus, H.-D.; Hermes, H.; Hirzebruch, F.; Koecher, M.; Mainzer, K.; Neukirch, J.; Prestel, A.; Remmert, R. (1991) Graduate Texts in Mathematics-Readings in Mathematics, Springer Verlag, Berlin. 
[5] Fischler, H.; Lichtfeldt, M. (1991) "Learning quantum mechanics", in: R. Duit, F. Goldberg, \& H. Niedderer (Eds.) Research in Physics Learning: Theoretical Issues and Empirical Studies, IPN, Kiel.

[6] Fischler, H.; Lichtfeldt, M. (1992) "Modern physics and students' conceptions", Int. J. Sci. Educ. 14: 181-190.

[7] Greca, I.M.; Freire, O. (2014) "Teaching introductory quantum physics and chemistry: caveats from the history of science and science teaching to the training of modern chemists", Chem. Educ. Res. Pract. 15: 286-296.

[8] Griffiths, D.J. (1995) Introducción a la Mecánica Cuántica. Prentice Hall, Upper Saddle River NJ.

[9] Ireson, G. (2000) "The quantum understanding of pre-university physics students", Phys. Educ. 35: 15-21.

[10] Johnston, I.D.; Crawford, K.; Fletcher, P.R. (1998) "Student difficulties in learning quantum mechanics", Int. J. Sci. Educ. 20: 427-446.

[11] Kauffman, L.H. (2001) Knots and Physics, 3rd edition. World Scientific Publishing Company.

[12] Kronsbein, J. (1967) "Kinematics quaternions spinors and Pauli's spin matrices", American Journal of Physics 35(4): 335-342.

[13] Kuhn, T. (1962) La estructura de las revoluciones científicas. Universidad de Chicago, Chicago.

[14] Mashhadi, A. (1996) "Students' conceptions of quantum physics", in: G. Welford, J. Osborne \& P. Scott Falmere (Eds.) Research in Science Education in Europe: Current Issues and Themes, London: 254-265.

[15] Müller, R.; Wiesner, H. (2002) "Teaching quantum mechanics on an introductory level”, Am. J. Phys. 70(3): 200-209.

[16] Naber G. (2003) The Geometry of Minkowski Spacetime: An Introduction to the Mathematics of the Special Theory of Relativity. Dover Publications.

[17] Niedderer, H.; Bethge, T.; Cassens, H. (1990) "A simplified quantum model: A teaching approach and evaluation of understanding", in: P.L. Lijnse et al. (Eds.) Relating Macroscopic Phenomena to Microscopic Particles: A Central Problem in Secondary Science Education, CD-b Press, Utrecht. 
[18] Petrache, H.I. (2014) "Coset group construction of multidimensional number systems”, Symmetry 6(3): 578-588 [arXiv:1212.1850].

[19] Petri, J.; Niedderer, H. (1998) "A learning pathway in high-school level atomic physics", Int. J. Sci. Educ. 20: 1075-1088.

[20] Rodríguez Bouza, V. (2012) "Sobre los cuaterniones, álgebras de Lie, y matrices de Pauli. Teoría básica y aplicaciones físicas”. Recuperado el 10 de febrero de 2016 de: http://dspace.sheol.uniovi.es/ dspace/handle/10651/18233

[21] Singh, C. (2001) "Student understanding of quantum mechanics", American Journal of Physics 69(8): 885-895.

[22] Staley, M. (2010) "Understanding quaternions and the Dirac belt trick", European Journal of Physics 31(3): 467.

[23] Styer, D.F. (1996) "Common misconceptions regarding quantum mechanics”, American Journal of Physics 64: 1202-1202.

[24] Torres, C. (1994) “Rotaciones y espinores (Rotations and spinors)”, Revista Mexicana de Física 40(1): 119-131.

[25] van der Waerden, B.L. (1976) Hamilton's discovery of quaternions Math. Mag. 49: 227-234.

[26] Zhu, G.; Singh, C. (2013) "Improving student understanding of addition of angular momentum in quantum mechanics", Physical Review Special Topics-Physics Education Research 9(1): 010101. 